\title{
Medicina genómica aplicada a la salud pública
}

\author{
Ana Burguete, Dra en C, ${ }^{(1)}$ Víctor H Bermúdez-Morales, M en C, ${ }^{(I, 2)}$ \\ Vicente Madrid-Marina, Dr en C. ${ }^{(I)}$
}

Burguete A, Bermúdez-Morales VH, Madrid-Marina V. Medicina genómica aplicada a la salud pública. Salud Publica Mex 2009;5 I supl 3:S379-S385.

\section{Resumen}

La genómica, visualizada como una disciplina científica encargada del mapeo, secuenciación y análisis de los genomas, ha facilitado la identificación y comprensión de las formas de organización y función de los genes de los organismos, lo cual ha generado un amplio conocimiento de la estructura y la función de los genomas. La influencia de la genómica en la medicina ha creado una nueva visión acerca de la forma de percibir los episodios patológicos y fisiológicos, tras conocer la influencia de las variaciones genéticas sobre la susceptibilidad a la enfermedad. En la salud pública, mediante la epidemiología genética, el conocimiento genético ha promovido acciones individuales y poblacionales para evaluar el efecto de la distribución de los determinantes genéticos y su interacción con factores ambientales en la etiología de las enfermedades humanas. De modo adicional, la medicina genómica propone nuevos sistemas de diagnóstico, relaciones genéticas y alteraciones alimenticias, respuesta específica a diversos medicamentos y diseño de nuevos fármacos para grupos susceptibles. Sin embargo, los grandes avances de la medicina genómica en el campo de la salud aún son sólo promisorios.

Palabras clave: genómica; epidemiología; salud pública; México
Burguete A, Bermúdez-Morales VH, Madrid-Marina V. An overview in genomic medicine and public health. Salud Publica Mex 2009;5 I suppl 3:S379-S385.

\begin{abstract}
Genomics, as a scientific discipline responsible for genome maps, sequencing and functional analysis of genomes, allows for continually expanding knowledge of the structure and function of genomes. The influence of genomics on medicine generates a new perspective for how we perceive health and disease, knowing the influence of genetic variations on susceptibility to disease. In the area of public health, genetic epidemiology translates genetic knowledge into individual and public actions, evaluating the effect of the distribution of genetic determinants and their interaction with environmental factors involved in the etiology of human diseases. In addition, genomic medicine suggests new diagnostic systems, genetic associations and nutritional disorders, specific responses to diverse drugs, and the design of new drugs for susceptible groups. And yet, the greatest advances in genomic medicine in the field of health are forthcoming.
\end{abstract}

Key words: genomic; epidemiology; public health; Mexico

(I) Dirección de Enfermedades Crónicas y Cáncer. Centro de Investigación sobre Enfermedades Infecciosas, Instituto Nacional de Salud Pública. Cuernavaca, Morelos, México.

(2) Facultad de Medicina, Universidad Nacional Autónoma de México. México, DF, México. 
T a genómica, visualizada como disciplina científica ـencargada del mapeo, secuenciación y análisis de los genomas, ha facilitado la identificación y la comprensión de la función de los genes. Esta ciencia se divide en estructural y funcional. La genómica estructural caracteriza la naturaleza física y expresión de los genomas completos; por su parte, la genómica funcional estudia los mecanismos de acción de los genes. ${ }^{1,2}$ En medicina, la genómica abre una nueva perspectiva para entender los procesos biológicos de salud y enfermedad e influye de manera directa acerca de cómo percibir las distintas afecciones. ${ }^{3}$ Las variaciones del genoma y su relación con la enfermedad son claves muy importantes para entender, diagnosticar, tratar y quizá incluso prevenir algunas enfermedades. ${ }^{4}$ De esta manera, la medicina genómica permitirá la reclasificación de las anomalías y algún día creará una nueva y mejor práctica clínica, más predictiva y personalizada (cuadro I). ${ }^{5-7}$

En virtud de la amplitud del campo de la genómica, que incluye disciplinas relacionadas como la trascriptómica, la proteómica y la epigenómica, entre otras más, en esta revisión sólo se alude a todo aquello relacionado con elDNA, tal y como se encuentra en el genoma, incluidos polimorfismos, mutaciones, inserciones y deleciones de material genético.

\section{El genoma humano}

El genoma humano está organizado en 23 pares de cromosomas, que contienen cerca de 30000 genes que codifican entre 250000 y 1000000 de proteínas ${ }^{6}$ contenidas en 100 millones de pares de bases. ${ }^{7,8}$

El código genético depende de combinaciones de tres de los nucleótidos que forman la hebra del ADN. Éstos, adenina (A), citosina $(\mathrm{C})$, guanina $(\mathrm{G})$ y timina (T), dan lugar a los aminoácidos, que especifican todas las propiedades biológicas de las proteínas, de tal forma que conocer la secuencia de un gen predice la secuencia de la proteína y su función biológica. Un cambio en la secuencia de nucleótidos, por sutil que sea, puede tener un significado funcional trascendental que puede

\section{Cuadro I \\ LA MEDICINA PERSONALIZADA}

Prevención-diagnóstico-tratamiento individual

- Predicción genética de sensibilidad a enfermedades.

- Análisis de las características moleculares concretas de la enfermedad (diagnóstico molecular).

- Predicción de sensibilidad/ resistencia a tratamientos y a sus efectos adversos, en función de las características del paciente y la enfermedad.

- Diseño de una terapia específica en función de los datos anteriores. predisponer a una enfermedad; ${ }^{9}$ esto es de particular importancia en las enfermedades complejas y no así en aquellas que tienen un patrón de herencia mendeliano dominante o recesivo.

Uno de los mayores retos en el estudio de las enfermedades complejas ha sido la generación de perfiles genómicos que permitan establecer la relación entre los cambios genéticos y el comportamiento clínico y terapéutico. Esta relación de la enfermedad puede servir para caracterizar marcadores pronósticos de susceptibilidad a enfermedad, que sirvan como respuestas predictivas para distintas modalidades terapéuticas o bien ser blanco de terapias futuras. ${ }^{10,11}$ Los estudios de la medicina genómica aportan la posibilidad de encontrar un locus que identifique la "heredabilidad" en enfermedades complejas, tanto en las características propias de un gen (polimorfismos), como cuantitativamente al considerar número de copias, amplificaciones $\mathrm{y}$ deleciones del gen.

Los polimorfismos de un solo nucleótido o SNP (por sus siglas en inglés single nucleotide polymorphism) son variaciones en la secuencia del $\mathrm{ADN}$ caracterizadas por cambios de un solo nucleótido (A, T, C o G) a lo largo del genoma. ${ }^{12-14}$ Los SNPs ocurren con una periodicidad aproximada de cada 100 o 300 bases a lo largo de los 3000 millones de bases del genoma humano y proporcionan 90\% de la variabilidad genética humana. Los SNPs aparecen en regiones codificantes y no codificantes del genoma, son bialélicos, relativamente estables al paso del tiempo y se transmiten de una generación a otra. ${ }^{12-14}$

Las variaciones en la secuencia de nucleótidos presentes en el ADN pueden tener un gran impacto sobre la forma en que los seres humanos responden a las agresiones ambientales, entre ellas agentes infecciosos, toxinas, compuestos químicos, componentes de la dieta y fármacos. En consecuencia, el estudio de la distribución de los SNPs en genes definidos del genoma en ciertas poblaciones específicas es fundamental para comprender los procesos biológicos, las enfermedades y la respuesta a los agentes terapéuticos. Debido a que las regiones no codificantes del genoma contienen múltiples secuencias reguladoras, los estudios de genómica no deben limitarse a la búsqueda de estos posibles marcadores en genes codificantes ( $2 \%$ del genoma).$^{15}$

El Proyecto Internacional de Mapeo de Haplotipos (HapMap) ${ }^{16}$ consiste en bases de datos públicas que contienen hoy día más de dos millones de cambios de bases como marcadores con frecuencias alélicas verificadas. Hasta la fecha se han reportado cerca de tres millones de SNPs relacionados con distintas enfermedades, sobre todo de tipo monogénico. ${ }^{16}$ Sin embargo, como ya se mencionó, en enfermedades complejas no mendelianas, como las enfermedades cardiovasculares 
(arterioesclerosis), oncológicas, nutricionales (diabetes, obesidad), degenerativas (p. ej., Parkinson y Alzheimer), autoinmunitarias (p. ej., esclerosis múltiple, artritis reumatoide), alérgicas (asma) y otras, su estudio es difícil por la gran variedad de defectos genéticos vinculados con penetrancia variable en virtud de la compleja combinación de genes de susceptibilidad y la influencia de factores externos en su desarrollo; ${ }^{16,17}$ por consiguiente, se necesitan estudios de gran escala efectuados en poblaciones específicas para la identificación de los genes de susceptibilidad relacionados con diferentes anomalías. ${ }^{18}$

\section{Genómica y epidemiología}

La genómica en salud pública evalúa el efecto y la interacción de los genes y el ambiente sobre la salud de la población. Por lo regular, los epidemiólogos estudian la relación entre el ambiente (entendido en sentido amplio) y la aparición de enfermedades en el ser humano. Por otro lado, los investigadores del campo de la genética poblacional evalúan los efectos de la estructura de la población y las fuerzas de la selección sobre la frecuencia de los rasgos genéticos..$^{19,20}$

La epidemiología genética combina el método genético con el epidemiológico para analizar la variación genética en poblaciones humanas y su relación con las variaciones fenotípicas normales y patológicas. En este contexto, los epidemiólogos genéticos evalúan la distribución y los determinantes de los rasgos genéticos en poblaciones humanas y describen la función de los factores genéticos y su interacción con factores ambientales en el origen de las enfermedades humanas. ${ }^{20,21}$ Por lo tanto, para definir la epidemiología genética deben abarcarse todos los aspectos de la genética poblacional, excepto la evolución, incluidos el fenotipo, las interacciones genético-ambientales y los modos de transmisión relacionados con la salud o la ubicación de los genes que provocan enfermedades. ${ }^{20-21}$ Este nuevo abordaje epidemiológico de la genómica plantea la posibilidad para detectar poblaciones en riesgo, tras combinar los factores de riesgo convencionales y los genéticos; es decir, este análisis genético puede usarse para identificar a los individuos que tienen riesgos genéticos adicionales y en quienes la reducción de otros factores de riesgo no genéticos resultaría beneficiosa como una herramienta preventiva de enfermedad. ${ }^{22}$

Las herramientas genómicas son determinantes para el diseño de estudios epidemiológicos, el análisis y la inferencia causal de factores ambientales vinculados con la enfermedad. ${ }^{22,23}$ Existen diferentes conductas de estudio que tienen como objetivo identificar un polimorfismo o variante en un gen relacionado con una afección.
Para obtener evidencia de que una enfermedad posee una base genética, se utilizan los estudios de agregación familiar o de gemelos. Para localizar los genes de interés para un determinado padecimiento, se realizan estudios denominados de ligamiento (linkage), que emplean como marcadores genéticos una serie de polimorfismos repartidos a lo largo del genoma. En estos estudios se utilizan familias grandes con varios miembros afectados y ello hace posible identificar zonas del genoma de interés. ${ }^{23,25}$ Para identificar con mayor precisión los genes de interés afectados, se conducen estudios de asociación genética, en los que se compara la frecuencia relativa de las diferentes variantes polimórficas de los genes candidatos entre los individuos afectados y un grupo control adecuado. ${ }^{24,26,28}$

Diversos diseños epidemiológicos basados en individuos no relacionados pueden emplearse para conducir estudios de asociaciones genéticas. Los estudios de casos y controles son los más usados para estudiar los determinantes de mutaciones humanas, evaluar el papel que desempeñan los indicadores genéticos inespecíficos (consanguinidad y mezcla de razas) en el origen de las enfermedades y determinar la función etiológica de los rasgos genéticos. ${ }^{27,28}$ También se pueden utilizar diseños basados en familias, en los cuales los individuos control son parientes de los casos, como los diseños de casos y hermanos sanos o tríos (caso y padres). ${ }^{28,30} \mathrm{Un}$ estudio de asociación genética se diseña para determinar la relación que existe entre uno o más marcadores genéticos y la frecuencia o gravedad de un suceso en particular. Estos protocolos son similares a los estudios de asociación entre variables demográficas, ambientales, características clínicas y una enfermedad en particular, con la diferencia de que en vez de explorar, por ejemplo, el efecto de la edad, el nivel de colesterol y su relación con una enfermedad coronaria, la característica bajo estudio es un marcador genético, en relación con un trastorno o fenotipo. ${ }^{30,31}$

Una de las grandes aportaciones de la epidemiología genética en la salud radica en evaluar el efecto terapéutico de un fármaco frente a una enfermedad y cómo éste depende de la variabilidad interindividual de la respuesta a dicho fármaco. Esta variabilidad se vincula de manera intrínseca con las características genéticas del sujeto reguladas por factores fisiológicos, patológicos y ambientales, además de una particular dotación genética del individuo. En esta dotación genética subyacen factores farmacocinéticos determinantes de la concentración del fármaco en su lugar de acción y en los factores farmacodinámicos (acción específica del fármaco) ligados a la manifestación propia de la enfermedad y la reacción adversa de éste. ${ }^{32}$ Por lo tanto, la farmacología del futuro realizará una terapéutica individualizada tras 
vigilar el cociente beneficio-riesgo en los sujetos; es decir, determinará el fármaco de elección para la manifestación específica de la enfermedad en la persona y la dosis apropiada para conseguir el efecto terapéutico, con un riesgo de reacciones adversas mínimo. ${ }^{32}$

Aunque los términos farmacogenética y farmacogenómica se han utilizado con frecuencia de manera indistinta, en la actualidad existe ya una opinión unánime para referirse a la farmacogenética como el estudio de las bases genéticas que modifican la respuesta individual a los fármacos; esto es, estudia la estirpe entre genotipos individuales y la capacidad de metabolizar un compuesto. Por su parte, la farmacogenómica analiza las aplicaciones de la tecnología genómica en el desarrollo de fármacos y terapéuticas mediante la detección y vigilancia del tratamiento de la causa molecular de las enfermedades. ${ }^{33,34}$ Ambas disciplinas comparten muchos puntos en común; por ejemplo, la búsqueda de fármacos más específicos para la enfermedad (un objetivo de la farmacogenómica) pasa por la localización de blancos terapéuticos que la farmacogenética proporciona. El desarrollo de los métodos de análisis masivos de SNPs permitirá asignar a cada individuo un "perfil de SNPs" que recoja información sobre diversas áreas del genoma relacionadas con la eficacia y la tolerancia a diversos agentes terapéuticos. Además, se han descubierto genes relacionados con las diferencias de respuesta frente a los fármacos. ${ }^{6,34-38}$ Una enfermedad progresiva que desarrolla resistencia a los tratamientos podrá tratarse con terapias alternativas dirigidas a blancos específicos participantes en los mecanismos de resistencia. ${ }^{7}$

Por otro lado, el desarrollo genómico hará posible conocer con precisión de qué modo algunos factores ambientales, como los componentes de la dieta, influyen de forma notoria en los patrones de expresión de la información genética, además de alteraciones patológicas, lo cual se basa en la constitución genética de las personas. Esta nueva era de la nutrición molecular (interacciones genes-nutrimentos) posee dos áreas esenciales: la nutrigenómica, que estudia la influencia de los nutrimentos sobre la expresión de genes, y la nutrigenética, que evalúa la influencia de las variaciones genéticas en la respuesta del organismo a los nutrimentos. ${ }^{35,39-42} \mathrm{La}$ primera suministra un conocimiento molecular sobre los componentes de la dieta que contribuyen a la salud mediante la alteración de la expresión o estructuras, según sea la constitución genética individual. ${ }^{40}$

Los principios de la nutrigenómica son: a) los componentes de la dieta directos o indirectos pueden alterar la expresión o estructura de los genes; b) la dieta puede ser un factor de riesgo de una enfermedad; c) algunos genes regulados por la dieta (y sus variantes comunes) pueden desempeñar una función en el inicio, incidencia, progresión o gravedad de las enfermedades crónicas; d) el grado en el cual la dieta influye sobre el binomio salud-enfermedad puede depender de la constitución genética individual; y e) cualquier intervención dietética basada en el conocimiento de las necesidades nutricionales, el estado nutricional y el genotipo (p. ej., «la nutrición individualizada ») será útil para prevenir, mitigar o curar las afecciones crónicas. ${ }^{40}$

Más aún, la nutrigenética engloba el análisis retrospectivo de las variantes genéticas de los individuos que inducen la respuesta clínica a los nutrimentos, lo cual permite la posibilidad de personalizar la nutrición de acuerdo con la constitución genética de los consumidores, sin perder de vista el conocimiento de las variantes genéticas que afectan al metabolismo de los nutrimentos y los blancos de los nutrimentos. El progreso de la nutrigenómica y la nutrigenética estará ligado al consumo de dietas personalizadas para evitar el inicio de la enfermedad y optimizar el mantenimiento de la salud humana. ${ }^{39,41,42}$

\section{La genómica y los sistemas diagnósticos}

Las pruebas diagnósticas basadas en la secuencia del ADN se consideran fundamentales en la aplicación médica en el último decenio. Dichas pruebas permitirán en un futuro muy cercano la identificación y caracterización de un elevado número de genes que ocasionan padecimientos humanos. Las pruebas genéticas pueden usarse para el diagnóstico de enfermedades, confirmarlo o proporcionar información pronóstica acerca del curso de alguna enfermedad. Asimismo, confirman la existencia de afecciones en individuos asintomáticos $y$, con un grado de precisión muy certero, predicen el riesgo de futuras anomalías en sujetos sanos o su progenie. ${ }^{43}$

Las estrategias típicas para el diagnóstico genético pueden clasificarse como directas o indirectas, según sea que detecten o no el gen. En el primer caso es posible determinar el diagnóstico tras identificar en los pacientes las diferentes mutaciones relacionadas con enfermedades genéticas raras que presentan patrones de herencia mendeliana. Estas anormalidades incluyen las distrofias miotónicas y muscular de Duchenne, fibrosis quística, neurofibromatosis tipo 1, anemia de células falciformes y enfermedad de Huntington. ${ }^{43}$

La segunda medida o método indirecto, independiente del conocimiento del gen causal, se fundamenta en el estudio de la herencia conjunta de diferentes marcadores y el locus de la enfermedad estudiada. Para este fin es preciso que el marcador utilizado muestre un sólido nexo con el locus de interés. ${ }^{43}$ Para aplicar el diagnóstico indirecto deben tenerse en cuenta los siguientes puntos: a) el gen causal del trastorno debe 
estar localizado; b) se requieren marcadores polimórficos informativos, intragénicos o adyacentes a dicho gen; c) son necesarias muestras de varios familiares (sanos y afectados) y no puede realizarse si tan sólo se dispone del caso índice; d) es imprescindible un diagnóstico clínico preciso de los familiares del caso índice; e) la recombinación genética entre el marcador y el gen puede reducir la probabilidad del resultado y f) en presencia de heterogeneidad genética debe analizarse el ligamiento con todos los posibles locus. ${ }^{43}$

Por otro lado, en la actualidad ya existen pruebas para detectar mutaciones para un puñado de afecciones más complejas, como el cáncer de mama, ovario y colon a nivel poblacional. Aunque estas pruebas tienen algunas limitaciones, pueden utilizarse para calcular el riesgo en individuos presintomáticos con antecedentes familiares de algún trastorno. De esta manera, el análisis molecular de biopsias es uno de los campos en los que ya se ha comenzado a trabajar; por ejemplo, mediante el empleo de los denominados "chips de ADN" se ha vigilado la expresión simultánea de una gran cantidad de genes en biopsias de pacientes con cáncer de mama o algunos tipos de linfoma o bien la detección del ADN del virus del papiloma humano. Mediante estas pruebas genéticas será posible reducir en grado considerable la incidencia de cáncer cervicouterino y en consecuencia disminuir la mortalidad por esta causa. ${ }^{44,45}$

La clasificación de los pacientes de acuerdo con sus patrones de expresión génica puede, de manera ulterior, correlacionarse con su pronóstico, con respuesta a determinadas terapias o bien con capacidad para generar metástasis del tumor primario. Un beneficio potencial de estas pruebas genéticas es que pueden suministrar información que ayude a los médicos y pacientes a controlar la enfermedad de forma más efectiva. ${ }^{44,45}$

\section{Genómica: vacunas y terapia génica}

Hace 35 años, el desarrollo de la biología molecular y la tecnología del ADN recombinante hizo posible avances en la investigación en la salud. Desde entonces estas tecnologías han develado lentamente los secretos moleculares de los genes y agentes infecciosos relacionados con la enfermedad. Sin embargo, la visión de la investigación en el campo de la salud ha dado saltos muy importantes con la genómica. No sólo se enfoca en la secuenciación de genomas, como ya se ha descrito, sino que permite una exhaustiva identificación de los genes candidatos, biomarcadores, vacunas y blancos antimicrobianos en favor de controlar las anomalías. 45,46

Durante el siglo pasado, varias técnicas se emplearon para el desarrollo de vacunas e incluyen el uso de bacterias vivas y virus atenuados, además de microorga- nismos muertos. Sin embargo, el objetivo principal del desarrollo de nuevas vacunas es la rápida identificación de los antígenos altamente inmunogénicos y protectores. La genómica ha contribuido en sumo grado a proveer un nuevo impulso para el campo microbiano; la secuencia genómica completa de un patógeno humano representa un nuevo campo apenas explorado para la producción de nuevas vacunas y fármacos antimicrobianos. ${ }^{46,47}$

En la era posgenómica, basada en la disponibilidad de la secuencia genómica entera de un organismo, tres nuevas disciplinas de biología molecular han emergido: la genómica, el perfil transcripcional y la proteómica. Todas estas tecnologías tienen el potencial de acelerar los procesos de identificación de antígenos como blancos de vacunas, además de validar y extender el rango de los antígenos candidatos para las vacunas. El progreso de estas nuevas tecnologías ha llevado a la consolidación de la ciencia de la bioinformática para el tratamiento y la evaluación crítica de la gran cantidad de información generada. 48,49

Más todavía, la transferencia de genes o terapia génica posee un potencial para el tratamiento y aun para la curación de algunas afecciones genéticas y adquiridas, como el cáncer y el sida, mediante el uso de genes normales para reemplazar genes defectuosos o bien magnificar alguna función, como la reacción inmunitaria. Hasta la fecha, más de 500 protocolos de terapia génica se realizan alrededor del mundo y suponen la participación de unos 3500 pacientes. La mayoría (78\%) se lleva a cabo en Estados Unidos de América, seguido de Europa (18\%). Aunque muchos de los protocolos están enfocados en el tratamiento de diversos tipos de cáncer, algunos estudios comprenden también enfermedades multigénicas y monogénicas, así como padecimientos infecciosos y cardiovasculares. Los primeros protocolos de terapia génica se idearon para corregir enfermedades hereditarias. Trastornos bien definidos, como la deficiencia de la enzima desaminasa de adenosina o la enfermedad crónica granulomatosa, resultaron atractivos para los estudios clínicos debido a la posibilidad de corregir la deficiencia de un gen a través de la inserción de una copia normal del gen mutado o deficiente. ${ }^{50,51}$

El gen insertado se integra exactamente en el mismo sitio y se regula de igual manera al gen defectuoso. En la actualidad, esta medida se ha aplicado sólo a células totipotenciales embrionarias murinas y no están disponibles para la terapia génica somática. En el caso de las enfermedades hereditarias monogénicas, la terapéutica génica somática sirve como "terapia de sustitución". Por lo tanto, el éxito de esta forma está limitada por la vida media de las células genéticamente modificadas en el organismo. A menos que las células totipotenciales 
transfectadas con genes terapéuticos se autorenueven, esta modalidad terapéutica deberá repetirse a lo largo de la vida del paciente.

En contraste con las enfermedades hereditarias, caracterizadas por defectos monogénicos bien definidos, la mayoría de los cánceres humanos se desarrolla en procesos de múltiples pasos que implican alteraciones de varios genes. La introducción de una copia de un gen supresor de tumores en células tumorales puede prevenir o revertir el fenotipo maligno in vitro..$^{52,53} \mathrm{Sin}$ embargo, esta alternativa no ha tenido éxito cuando se aplica a la clínica, ya que todas las células malignas deben transfectarse con una copia normal del gen con este procedimiento, mientras que las células normales deben protegerse. Este nivel de eficacia y especificidad aún no se logra con el estado actual de la tecnología de la terapia génica.

Hoy en día, los protocolos de terapia génica en oncología usan diferentes estrategias, como la inserción de genes de citocinas, los cuales pueden al parecer aumentar la reacción inmunitaria de los pacientes, ${ }^{54,55} \mathrm{y}$ la inserción de genes suicidas, los cuales pueden matar de forma selectiva a las células tumorales transducidas mediante la activación de un mecanismo autotóxico. De manera adicional, existen una gran variedad de medidas prometedoras que están en evaluación preclínica, como el uso de los oligonucleótidos antisentido y las ribozimas específicas para determinado gen, con el fin de bloquear la expresión de un gen particular. ${ }^{55,56}$

En fecha reciente se ha aplicado otra forma de interrupción de la síntesis de proteínas, mediante los ARN de interferencia (ARNi) ${ }^{57}$ En consecuencia, la terapia génica aún enfrenta el reto de superar muchos obstáculos científicos antes de convertirse en una práctica médica común para el tratamiento de las enfermedades humanas. ${ }^{57}$

\section{Conclusiones}

La misión y los objetivos de la genómica son promover la incorporación de los hallazgos y descubrimientos de la ciencia genómica a la práctica de la salud pública. Se concentran en la función que cumple la salud pública para mejorar las intervenciones diseñadas, diagnosticar, prevenir y controlar las principales enfermedades crónicas, infecciosas, ambientales y ocupacionales, lo que permitirá como efecto reducir o eliminar inequidades sociales. Por último, esto repercutirá en una mejor práctica médica personalizada. Para lograr esto es preciso crear conciencia y educar a profesionales en salud pública, proveedores de cuidados de salud, tomadores de decisiones y público en general respecto del papel que desempeña la nueva ciencia genómica en la salud.

\section{Referencias}

I. Hocquette JF.Where are we in genomics.J Physiol Pharmacol 2005;56(3):37-70.

2. Eggen A. Basics and tools of genomics. Outlook Agr 2003;4:215-2I7.

3. Collins FS, Green ED, Guttmacher AE, Guyer MS.A vision for the future of genomics research. Nature 2003;422:835-847.

4. Cooper RS, Psaty BM. Genomics and medicine: distraction, incremental progress, or the dawn of a new age? Ann Intern Med 2003;138:576-580.

5. Bell J. Predicting disease using genomics. Nature 2004;429:453-456.

6. Burton PR, Tobin MD, Hopper JL. Key concepts in genetic epidemiology. Lancet 2005;366:941-95I. Review. Erratum in: Lancet 2006;7;3:28.

7. Joshi VA, Kucherlapati R. Genetics and genomics in the practice of medicine. Gastroenterology 2008; 134:1284- 288.

8. Emilien G, Ponchon M, Caldas C, Isacson O, Maloteaux JM. Impact of genomics on drug discovery and clinical medicine. Q J Med 2000;93: $391-423$.

9. Collins F. Genetics: an explosion of knowledge is transforming clinical practice. Geriatrics 1999;54:41-47.

10. Genome-wide association study of 14,000 cases of seven common diseases and 3,000 shared controls. Nature 2007;447(7| 45):66I-678. II. Huang BE,Amos Cl, Lin DY. Detecting haplotype effects in genomewide association studies. Genet Epidemiol 2007.

12. Stephens JC. Single-nucleotide polymorphisms, haplotypes, and their relevance to pharmacogenetics. Mol Diagn 1999;4:309-317.

13. Madsen BE,Villesen P,Wiuf C.A periodic pattern of SNPs in the human genome. Genome Res 2007.

I4. Wiltshire S, de Bakker PI, Daly MJ.The value of gene-based selection of tag SNPs in genome-wide association studies. Eur J Hum Genet 2006; I4(II):|209-12/4.

15. Hirschhorn JN, Lindgren CM, Daly MJ, et al. Genomewide linkage analysis of stature in multiple populations reveals several regions with evidence of linkage to adult height. Am J Hum Genet 200I; 69(I):I06-II6. 16. Judson R, Salisbury B, Schneider J,Windemuth A, Stephens JC. How many SNPs does a genome-wide haplotype map require? Pharmacogenomics 2002;3(3):379-391.

17. Hirschhorn JN, Lohmuerller K, Byrne E, Hirschhorn K.A comprehensive review of genetic association studies. Genet Med 2002;4:45-61.

18. McKusickVA. The anatomy of the human genome: a neo-vesalian basis for medicine in the 21 st century. JAMA 200।;286:2289-2295.

19. Bobrow M. What have we learned from genomics? Miami Nat Biotechnol Short Rep 2003;14:45-46.

20. Khoury MJ, Millikan R, Little J, Gwinn M. The emergence of epidemiology in the genomics age. Int J Epidemiol 2004;33:936-944.

21. Khoury M, Beaty T, Cohen B. Fundamentals of genetic epidemiology. New York: Oxford University Press, 1993.

22. Khoury MJ,Yang Q. The future of genetic studies of complex human diseases: an epidemiologic perspective. Epidemiology 1998;9:350-354.

23. Morton NE. The future of genetic epidemiology. Ann Med 1992;24:557-562.

24. Iniesta R, Guinó E, Moreno V.Análisis estadístico de polimorfismos genéticos en estudios epidemiológicos. Gac Sanit 2005; 19(4).

25. Teare MD, Barrett JH. Genetic linkage studies. Lancet 2005;366: 1036-1044

26. Khoury MJ, Beaty-Tewi H.Aplicaciones del método de casos y controles en epidemiología genética. Bol Oficina Sanit Panam I996: I2I(5). 27. Caporaso N, Rothman N,Wacholder S. Case-control studies of common alleles and environmental factors. J Nat Can Inst Mon 1999;26. 28. Pritchard JK, Donnelly P. Case-control studies of association in structured or admixed populations. Theor Popul Biol 200I;60:227-223. 29. Cardon LR, Bell JI.Association study designs for complex diseases. Nat Rev Genet 2001;2:91-9. 
30. Gauderman WJ,Witte JS, Thomas DC. Family-based association studies. J Natl Cancer Inst Monogr 1999;26:3I-37.

3I. Cordell HJ, Clayton DG. Genetic association studies. Lancet 2005,366:||2|-||3|.

32. Evans WE. Pharmacogenomics - drug disposition, drug targets, and side effects. N Engl J Med 2003;348:538-549.

33. Lares-Asseff I,Trujillo-Jiménez F. La farmacogenética y su importancia en la clínica. Gac Med Mex 200I;I37(3).

34. Colhoun HM, Mckeigue P, Bailey, DS. Pharmacogenomics - it's not just pharmacogenetics. Curr Opin Biotech 1998:9(6);595-601.

35. Kaplan J. Genomics and medicine: hopes and challenges. Gene Ther 2002;9:658-66l.

36. Liao G, Zhang X, Clark DJ, Peltz G.A genomic "roadmap" to "better" drugs. Drug Metab Rev 2008;40:225-239.

37. Nebert DW, Zhang G,Vesell ES. From human genetics and genomics to pharmacogenetics and pharmacogenomics: past lessons, future directions. Drug Metab Rev 2008;40:187-224.

38. Gurwitz D. Education: teaching pharmacogenomics to prepare future physicians and researchers for personalized medicine. Trends Pharmacol Sci 2003;24:122-125.

39. Ordovas JM, Corella D. Nutritional genomics. Annu Rev Genomics Human Genet 2004;5:7I-I I8.

40. Corella D, Ordovas JM. Single nucleotide polymorphisms that influence lipid metabolism: Interaction with dietary factors. Annu Rev Nutr 2005;25:34I-390.

41. Marti A, Moreno-Aliaga MJ, Zulet A, Martinez JA. Advances in molecular nutrition: nutrigenomics and/or nutrigenetics. Nutr Hosp 2005;20(3): 157-164.

42. Gillies PJ. Nutigenomics: the rubicon of molecular nutrition. JAm Diet Assoc 2003; 103 Suppl. 2:S50-S55.

43. Barton JH. Emerging patent issues in genomic diagnostics. Nat Biotechnol 2006;24:939-94I.

44. Service RF. Recruiting genes, proteins for a revolution in diagnostics. Science 2003:300(56|7):236-239.

45. Weinstock GM. Genomics and bacterial pathogenesis. Genomics 2000;6(5).
46. Masignani V, Rappuoli R, Pizza M. Reverse vaccinology: a genome-based approach for vaccine development. Expert Opin Biol Ther 2002;2:895-905. 47. Chakravarti DN, Fiske MJ, Fletcher LD, Zagursky RJ. Mining genomes and mapping proteomes: identification and characterization of protein subunit vaccines. Dev Biol 2000;103:8I-90.

48. Chakravarti DN, Fiske MJ, Fletcher LD, Zagursky RJ. Application of genomics and proteomics for identification of bacterial gene products as potential vaccine candidates. Vaccine 2000;19:60I-612.

49. Moxon R, Rappuoli R. Bacterial pathogen genomics and vaccines. $\mathrm{Br}$ Med Bull 2002;62:45-58.

50.Anderson,WF. Human gene therapy. Science 1992;256:808-813.

5I. Baker SJ, Markowitz, S, Fearon ER, Willson JK, Vogelstein B. Suppression of human colorectal carcinoma cell growth by wild-type $\mathrm{p} 53$. Science 1990;249:912-915

52. Huang HJ, Yee JK, Chen PL, Bookstein R, Friedman T, Lee WH. Suppression of the neoplastic phenotype by replacement of the RB gene in human cancer cells. Science 1998;242: I563-I566.

53. Bermúdez-Morales VH, Peralta-Zaragoza O, Madrid-Marina V. Terapia génica con citocinas contra cáncer cervico uterino. Salud Publica Mex 2005;47:458-468.

54. Pace U, Bockman JM, Mackay B, Goldberg AR. Construction of ribozyme which descriminate between closely related mRNA's. In: Genetically targeted research and therapeutics: anti-sense and gene therapy. Colorado: Keystone, 1993:412.

55. Álvarez-Salas, LM, Arpawong TE, Dipaolo JA. Growth inhibition of cervical cells by anti-sense oligodeoxynucleotides directed to human papillomavirus type 16 E6 gene. Antisense \& Nucl Ac Drug Development 1999;9:441-450.

56. Sui G, Soohoo C,Aftar EB, Gay F, Shi Y, Forrester WC, et al.A DNA vector-based RNAi technology to suppress gene expression in mammalian cells. Proc Nat Acad Sci 2002;99:55 I5-5520.

57. Mountain A. Gene therapy: the first decade. Trends Biotech 2000;18:119-128. 